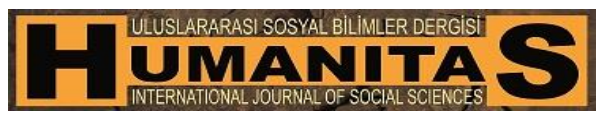

$\begin{array}{ll}\text { Humanitas, 2017; 5(10): 247-262 } & \text { http://humanitas.nku.edu.tr } \\ \text { ISSN: } 2147-088 X & \text { DOI: } \underline{10.20304 / \text { humanitas.292394 }}\end{array}$

Araştırma-İnceleme

\title{
QUELLE DEMARCHE SUIVRE EN DIDACTIQUE DE LANGUE ETRANGERE: LA GRAMMAIRE IMPLICITE OU LA GRAMMAIRE EXPLICITE?
}

\author{
Rıfat GÜNDAY ${ }^{1}$ \\ Fahriye ÇAKIR ${ }^{2}$ \\ Hasan ATMACA ${ }^{3}$
}

\begin{abstract}
Résumé: Il y a un rapport étroit entre la connaissance sur la langue et son emploi efficace. Sans connaître la grammaire d'une langue il est impossible de découvrir ses caractéristiques syntaxiques, morphologiques et sémantiques, et sans maîtriser une langue il est difficile de développer toutes les compétences langagières. Si on accepte la langue comme un système, pour comprendre et produire on a besoin de connaître les codes de ce système. Pour utiliser les éléments de la phrase à leurs places correctes, construire la communication exacte et pour s'exprimer à l'oral et surtout à l'écrit il est indispensable de savoir les emplois des règles de grammaire. Même si l'importance de l'enseignement de grammaire se diminue de temps en temps ou change d'une méthode à l'autre, la grammaire garde sa place incontournable en didactique de langue étrangère. Pour enseigner la grammaire de la langue étrangère il y a huit démarches: grammaire implicite et grammaire explicite, grammaire inductive et grammaire déductive, grammaire contextualisée et grammaire décontextualisée, grammaire active et grammaire passive. Il est possible aussi de classifier la grammaire selon les différentes catégories: grammaire linguistique et grammaire pédagogique. De plus, on peut analyser la grammaire en quatre dimensions: le lexique, l'orthographe, la phrase et le texte. Dans cette étude nous allons aborder la grammaire explicite et la grammaire implicite. La grammaire implicite, c'est l'enseignement de grammaire qui a le but de faire acquérir aux apprenants l'emploi de la langue cible sans avoir recours aux explications grammaticales et aux connaissances théoriques. Dans une telle démarche il s'agit d'un apprentissage intuitif. La grammaire explicite est une
\end{abstract}

\footnotetext{
${ }^{1}$ Prof. Dr., Ondokuz Mayıs Üniversitesi, Eğitim Fakültesi, Yabancı Diller Eğitimi Bölümü. rgunday@,omu.edu.tr

${ }^{2}$ Okt., Mersin Üniversitesi, Yabancı Diller Yüksekokulu. fahriyecakir@yahoo.fr

3 Arş. Gör., Ondokuz Mayıs Üniversitesi, Eğitim Fakültesi, Yabancı Diller Eğitimi Bölümü. hasan.atmaca@omu.edu.tr
} 
démarche dans laquelle on fait la description et l'explication des règles soit par le manuel et soit par l'enseignant. Ici, comme il s'agit de connaître explicitement les règles on peut parler d'un apprentissage cognitif. En outre, à partir des manuels de méthode en FLE nous allons aborder l'importance que la grammaire explicite et la grammaire implicite peuvent jouer au développement des compétences langagières. Notre étude est une recherche théorique. En étudiant des exemples et en prenant en considération les besoins des apprenants nous nous efforcerons atteindre un résultat: laquelle de deux démarches est plus efficace pour apprendre une langue étrangère?

Mots clés: Langue étrangère, Enseignement, Démarche, Grammaire explicite, Grammaire implicite

\section{YABANCI DİL ÖĞRETIMMINDE HANGİ YOL İZLENMELİ: AÇIK DÍLBÍLGÍsí Mİ ÖRTÜK DÍLBÍLGİsí Mİ?}

Öz: Dil üzerine bilgi ile dili etkin kullanma arasında sık1 bir bağıntı söz konusudur. Bir dilin dilbilgisini tanımadan onun söz dizimsel, yapısal ve anlamsal özelliklerini keşfetmek olanaksızdır ve bir dile yeterince hakim olmadan dil becerilerini geliştirmek güçtür. Şayet dil bir sistem olarak kabul edilirse, o dilde anlayabilmek ve üretebilmek için bu sistemin kodlarını tanımaya gereksinim duyulmaktadır. Cümlenin öğelerini yerlerinde kullanabilmek, doğru iletişimi kurabilmek ve kendini sözlü ve özellikle de yazılı ifade edebilmek için dilbilgisi kurallarının kullanımını bilmek kaçınılmazdır. Dilbilgisi öğretiminin önemi zaman zaman azalsa ya da bir yöntemden diğerine değişse de dilbilgisi yabancı dil öğretimindeki vazgeçilmez yerini korumaktadır. Hedef dilin dilbilgisini öğretmek için sekiz farklı yol izlenmektedir: örtülü ve açık dilbilgisi, tümevarım yoluyla ve tümden gelim yoluyla dilbilgisi, bağlamsal ve bağlam dışı dilbilgisi, etken ve edilgen dilbilgisi. Ayrıca dilbilgisi, sözcük, yazım, cümle ve metin boyutunda da ele alınabilir. Bu çalışmada açık dilbilgisi ve örtük dilbilgisi konusunu ele alacağız. Örtük dilbilgisi öğretimi, kuramsal bilgilere ve dilbilgisi açıklamalarına başvurmadan hedef dilin kullanımını öğrenenlere kazandırmayı hedeflemektedir. Böyle bir yol izlendiğinde sezgisel bir öğrenim söz konusudur. Açık dilbilgisi öğretiminde ise ders kitabı veya öğretmen tarafından kuralların betimlenmesi ve açıklanması şeklinde bir yol izlenmektedir. Burada kuralları açık bir şekilde tanıma olanağı sunulduğu için bilişsel bir öğrenme söz konusudur. Ayrıca bu çalışmada açık dilbilgisi ve kapalı dilbilgisinin dil becerilerinin geliştirilmesinde oynadığı rolü irdeleyeceğiz. Sonuç olarak yabancı dil öğreniminde hangi yolla izlemenin daha etkili olduğu sorusuna yanıt arayacağız.

Anahtar Sözcükler: Yabancı Dil, Öğretim, Yol/İşleyiş, Açık Dilbilgisi, Örtük Dilbilgisi

\section{Introduction}

Qu'est-ce que c'est la grammaire? D'abord, nous voulons commencer notre étude par la définition de la grammaire. Selon Robert (2015, p.499) la grammaire est «l'ensemble des règles à suivre pour parler et écrire 
correctement une langue.» Nous pouvons définir la grammaire en tant qu'un domaine scientifique qui étudie les règles de composition de la langue du point de vue syntaxique, morphologique, orthographique et lexicale.

Il y a un rapport étroit entre la connaissance sur la langue et son emploi efficace. Sans connaître la grammaire d'une langue il est impossible de découvrir ses caractéristiques syntaxiques, morphologiques et sémantiques, et sans maîtriser une langue il est difficile de développer les compétences langagières surtout au niveau avancé.

$\mathrm{Si}$ on accepte la langue comme un système, pour comprendre et produire on a besoin de connaître les codes de ce système. Sans la connaissance de vocabulaire et de grammaire, il est difficile d'acquérir des compétences orales et écrites (Bozbeyoğlu, 2002, p.87). Pour utiliser les éléments de la phrase à leurs places correctes, construire la communication efficace et pour s'exprimer à l'oral et surtout à l'écrit il est indispensable de savoir les emplois des règles de grammaire (Günday, 2015, p.192). Quand les apprenants ont maîtrisé les énoncés et les structures du système linguistique, ils peuvent les réemployer facilement et convenablement dans les situations différentes (Fougerouse, 2001, p.3). De plus, la connaissance des règles donne le sentiment de l'autonomie aux apprenants et augmente leur motivation envers l'emploi de la langue cible.

Dans l'enseignement de langue étrangère la grammaire est l'une de trois composantes langagières (grammaire, lexique, phonétique) et considérée comme un outil/instrument à l'acquisition de quatre compétences principales (compréhension orale, compréhension écrite, production orale, production écrite).

Même si l'importance de l'enseignement de grammaire se diminue de temps en temps d'une méthode à l'autre dans l'histoire de la méthodologie, la grammaire garde sa place incontournable en didactique de langue étrangère.

Mais les discussions au sujet de l'enseignement de grammaire continuent. L'enseignement de grammaire doit-il être effectué comment? Pour enseigner la grammaire de la langue étrangère il y a huit démarches: grammaire implicite et grammaire explicite, grammaire inductive et grammaire déductive, grammaire contextualisée et grammaire décontextualisée, grammaire active et grammaire passive.

Il est possible aussi de classifier la grammaire selon les différentes catégories: grammaire linguistique et grammaire pédagogique. Alors que la grammaire linguistique traite des connaissances grammaticales et des modèles théoriques, la grammaire pédagogique est au service de l'apprenant en vue d'aider sa compréhension en expliquant le fonctionnement des règles d'emploi de la langue cible.

Dans cette étude nous allons aborder la grammaire explicite et la grammaire implicite et nous allons considérer l'importance que la grammaire implicite et la grammaire explicite peuvent jouer au développement des compétences langagières et dans le bon usage de la langue étrangère. En outre, à partir des 
manuels de méthode en FLE nous allons aborder l'importance que la grammaire explicite et la grammaire implicite peuvent jouer au développement des compétences langagières. Notre étude est une recherche théorique. En étudiant des exemples et en prenant en considération les besoins des apprenants nous nous efforcerons atteindre un résultat: laquelle de deux démarches est plus efficace pour apprendre une langue étrangère ?

\section{Les catégories de grammaire}

Quand on étudie le sujet de grammaire il est important aussi de connaître les différents types de grammaire et les subdivisions, la corrélation entre eux et la place que chacun occupe dans l'enseignement/apprentissage d'une langue étrangère.

On peut aborder la grammaire en deux catégories: grammaire linguistique (descriptive) et grammaire pédagogique (prescriptive). La dernière se divise en sous-catégories comme grammaire d'apprentissage (la grammaire qui se présente sous forme de nombreux manuels mis entre les mains des apprenants), grammaire d'enseignement (les programmes grammaticaux destinés principalement aux enseignants) et grammaire de référence (un point de jonction entre grammaire pédagogique et grammaire linguistique). (Germain et Seguin, 1995, p.1; Mahommed, 2008)

Selon Mahommed (2008), la grammaire linguistique vise avant tout à décrire et donc à expliquer la connaissance grammaticale sans tenir compte du contexte réel d'emploi de la langue. En même temps elle traite aussi les grammaires de référence.

Quant à la grammaire pédagogique, elle «porte sur toute description des règles grammaticales qui aide l'apprenant à apprendre une langue et qui sert l'enseignant à expliquer le fonctionnement des règles d'emploi et d'usage de la langue enseignée. Donc, la grammaire pédagogique représente une mise en pratique concrète, par les pédagogues et les didacticiens, de la compétence grammaticale en s'appuyant sur la grammaire linguistique... Dans ce type de grammaire l'accent est mis sur les situations concrètes interpersonnelles et sur les emplois réels de la langue » (Mahommed, 2008). Ce type de grammaire ne contient pas un modèle théorique.

\section{La grammaire aux dimensions différentes}

On peut analyser la grammaire en quatre dimensions : le lexique, l'orthographe, la phrase et le texte.

A la dimension (Au niveau) de lexique, on peut étudier la formation des mots ; analyser la base, la racine, la terminaison, le préfixe, le suffixe.

A la dimension d'orthographe grammaticale on peut traiter la conjugaison.

A la dimension de grammaire de phrase, on aborde chaque élément qui compose la phrase. En didactique de grammaire on fait en général des explications sur les règles à ce niveau, c'est la partie la plus complexe où on rencontre les difficultés. 
A la dimension de grammaire de texte, on peut analyser le discours, les notions, les connecteurs et les rapports entre les éléments et les phrases dans l'unité du texte.

Pourtant malgré certaines ressemblances, même si les langues viennent de la même famille, chaque langue dispose des règles propres à soi.

\section{Les options dans l'enseignement de grammaire}

Pour enseigner la grammaire (morphosyntaxe) de la langue étrangère il existe des options différentes. Trois options sont utilisées (Besse, 1992, pp.22-23):

1) On donne des explications et on formule progressivement des règles. Pour la première option, il s'agit toujours d'apprendre une description morphosyntaxique de la langue étrangère. On suit plutôt une démarche déductive (de la règle explicitée aux exemples qui l'illustrent). On peut parler d'un enseignement traditionnel et de la grammaire explicite.

2) On refuse de donner des explications grammaticales, mais on fait pratiquer aux apprenants, de manière intensive, des exercices systématiques (comme les exercices structuraux). Ils leur permettent, par la répétition d'analogies de construction, de fixer les régularités morphologiques et syntaxiques de la langue étrangère. On préfère plutôt une démarche inductive (des exemples répétés, de la manière intensive à la règle implicite). Malgré tous ces exercices systématiques pour la seconde option il est possible de parler d'une grammaire implicite.

3) On refuse les explications et les exercices formels et on suit des procédures comme donner des exemples, des activités, des tâches à effectuer, des jeux de rôle, etc qui impliquent une pratique guidée de la langue étrangère, mais sans spéculer des repérages grammaticaux précis. Dans ce cas on apprend la grammaire dans et par la communication et l'action. La troisième option ne cherche pas à enseigner une description grammaticale de la langue étrangère. Elle vise, par diverses simulations et pratiques, à en faire intérioriser les régularités. Pour la troisième option il faut parler de la grammaire implicite.

\section{La grammaire implicite et la grammaire explicite dans les méthodes}

Dans la méthode grammaire-traduction (la méthode traditionnelle) on accorde une large place à l'enseignement de grammaire, il s'agit de l'enseignement d'une grammaire explicite et une grammaire descriptive. Ici, on cherche la réponse à la question de grammaire du comment et de grammaire du pourquoi. On analyse la structure linéaire de la langue étrangère, autrement dit sa structure morphosyntaxique. Ce qui est essentiel, c'est de découvrir les lois et les principes qui constituent le dynamisme intérieur d'une langue.

La méthode directe ne met pas la grammaire au centre de l'enseignement de langue étrangère. Elle est contre les descriptions de grammaire que la méthode traditionnelle (la méthode grammaire-traduction) leur donnait la priorité. Dans l'enseignement on peut parler d'une grammaire implicite. 
Pour la méthode audio-orale les exercices structuraux occupent une place importante du point de vue de grammaire. Mais on ne fait pas les analyses des règles de grammaire. A partir des pratiques d'exemples similaires on vise à enseigner la structure de la langue cible. On aperçoit une grammaire implicite.

La méthode audio-visuelle suit une démarche parallèle à celle de la méthode directe en didactique de la grammaire. Du fait que la priorité est accordée à l'acquisition des compétences orales et du vocabulaire, l'enseignement de la grammaire n'a pas une place considérable et il s'agit d'une grammaire implicite.

Bien que la méthode communicative accorde la priorité à la communication et au développement de quatre compétences langagières, dès le niveau débutant elle ne renonce pas à l'enseignement de grammaire, car selon cette méthode la grammaire est un moyen important en vue de développer ces compétences. En général, cette méthode préfère la grammaire implicite et inductive, mais parfois elle s'adresse aussi à la grammaire explicite. «L'enseignement de la grammaire pour ce courant doit être à la fois implicite dans les activités communicatives et explicites dans l'analyse réfléchie de la structure enseignée. C'est-à-dire, à partir des activités communicatives et des exercices qui favorisent la mise en situation de communication, on demande aux élèves de réfléchir pour déduire la règle de la structure grammaticale étudiée. » (Mahommed, 2008).

A l'heure actuelle, d'une part la méthode actionnelle met la co-action ou l'interaction au centre de l'enseignement de langue étrangère et de l'autre part, son objectif est de développer quatre compétences principales: compréhension orale, compréhension écrite, production orale et production écrite. Mais, en tant qu'une composante langagière, la grammaire joue un rôle considérable au développement de ces compétences. De même la méthode communicative, la méthode actionnelle suit généralement une démarche implicite et inductive, mais dans certaines situations s'il est nécessaire on fait aussi des explications, autrement dit on donne aussi la place à la grammaire explicite.

Alors que la méthode traditionnelle focalise l'enseignement de langue étrangère sur la grammaire, dans la méthode directe, la méthode audio-orale et la méthode audio-visuelle la grammaire perd sa place privilégiée. Après l'approche cognitive, d'une part pour la première fois on accorde la même importance au développement de quatre compétences langagières, et d'autre part, de nouveau, on accepte le rôle incontournable de la grammaire. Les deux dernières méthodes préfèrent un enseignement de grammaire fonctionnelle qui contient généralement une démarche implicite mais sans abandonner tout à fait des explications de grammaire.

\section{La grammaire implicite}

La grammaire implicite, c'est l'enseignement de grammaire qui a le but de faire acquérir aux apprenants l'emploi de la langue cible sans avoir recours aux explications grammaticales et aux connaissances théoriques. Mais «implicite ne veut pas dire "absent", mais plutôt "caché" d'une sorte que l'apprenant ne sent pas qu'il est en train d'apprendre une langue étrangère, ce qui n'est pas facile. » 
(Mahommed, 2008). Dans une telle démarche il s'agit d'un apprentissage intuitif.

Dans la démarche où on suit la grammaire implicite, l'enseignement/apprentissage de grammaire est effectué essentiellement sur les exercices (Vigner, 2008, p.65). Les exercices structuraux de la méthode audioorale constituent l'un des exemples de grammaire implicite. On pratique les exercices structuraux sur «la phrase modèle ». Grâce à ces exercices intensifs les apprenants apprennent automatiquement les règles de grammaire et ils peuvent les appliquer dans les situations similaires (Mahommed, 2008, p.9). Ces exercices développent la performance de la grammaire sans s'adresser aux explications, même s'ils restent restreints pour les situations très particulières. Dans les manuels de méthodes actuels il y a plusieurs activités et exercices afin d'enseigner la grammaire d'une manière implicite. Besse et Porquier (1993, p.86) proposent d'enseigner la grammaire d'une manière implicite. Ils remarquent l'utilisation inconsciente des règles grammaticales d'une langue donnée dans une communication quotidienne.

Certains chercheurs, écrivains de manuels et professeurs prétendent qu'on peut apprendre une langue étrangère sans qu'on ait besoin de réfléchir aux règles. Selon eux les apprenants peuvent apprendre implicitement, y compris automatiquement, les règles de grammaire de la langue cible telles qu'ils acquissent leur langue maternelle. Mais ce n'est pas possible en tout cas, car les deux processus sont très différents. Alors que l'acquisition de la langue maternelle se réalise inconsciemment dans la famille et dans la société, autrement dit dans un environnement social et authentique, l'apprentissage de la langue étrangère est un processus qui se développe consciemment en général dans un milieu scolaire et virtuel. Donc, les apprenants n'ont pas toujours, lors de l'apprentissage de langue étrangère, la possibilité de pratiquer la langue cible dans l'environnement authentique. Cette situation rend difficile la perception des règles de grammaire si on suit la démarche implicite.

Selon certains pédagogues «le but de l'enseignement de la grammaire est de maitriser le bon usage de la langue (avoir une compétence communicative) et non pas d'avoir forcement une connaissance grammaticale descriptive. Est-ce que la grammaire implicite est vraiment capable d'atteindre toute seule cet objectif ?» (Mahommed, 2008). Pour chaque cas ou utilisation ce n'est pas possible. Parce que les apprenants peuvent avoir du mal à saisir la finalité grammaticale de certains emplois ou structures. S'il faut donner un exemple :

A-Est-ce que les élèves pourront réussir à l'examen de français.

$B$ - J'en suis sûr.

A- Ils aiment le français.

$B$ - Je ne le sais pas 
Ici, sans expliquer l'expression «être sûr de », autrement dit sans avoir recours à la grammaire explicite, il est très difficile que les apprenants peuvent découvrir :

$$
\text { Verbe }+D E=E N / \text { Verbe }+\varnothing=L E \text { Exception. }
$$

Dans la grammaire implicite ce sont les apprenants qui doivent découvrir et formuler les règles. «Il faut plutôt adopter « l'approche par la découverte et l'ajustement progressif des règles » qui est fondée sur une théorie de type constructive.» (Mahommed, 2008). Donc, il est utile de «favoriser l'apprentissage grammatical à travers des interactions et discussions entre les élèves sur les règles de la langue. » (Courtillon, 1989, p.118)

Pour Chartrand (1996, p.398), «le fait de pratiquer un apprentissage scolaire de la L2, à visée communicative, sans mis accent expressément sur les connaissances grammaticales de la langue. » est une démarche idéale. Pourtant «il ne faut pas mettre l'accent sur la compétence communicative en ignorant complètement la grammaire, et non plus le contraire. Donc, il faut avoir une sorte d'équilibre entre ces deux extrêmes en s'appuyant toujours sur le fait que le but de l'enseignement d'une langue étrangère est de pouvoir communiquer dans une situation de communication réelle, et que la grammaire descriptive peut servir à accomplir cet objectif. Par contre, l'enseignement de la grammaire descriptive ne doit pas être imposé aux élèves d'une façon lourde.» (Mahommed, 2008).

Le manuel ou l'enseignant ne doit pas donner en détail l'explication sur la règle d'emploi. Car dans ce cas une grande partie de la durée peut être distinguée seulement pour l'enseignement de grammaire. Et cela peut retarder le développement des compétences principales.

Ici, nous voulons étudier un exemple tiré du manuel de méthode Alter Ego. C'est un livre composé à partir des principes de la méthode actionnelle, publié par la maison d'édition Hachette. Le niveau du manuel est A1 / le débutant. Voici un exemple de la partie «point langue» : 
Günday, R. ve Ark. (2017). Quelle Demarche Suivre en Didactique de Langue Etrangere:

La Grammaire Implicite ou La Grammaire Explicite? Humanitas, 5(10), 247-262

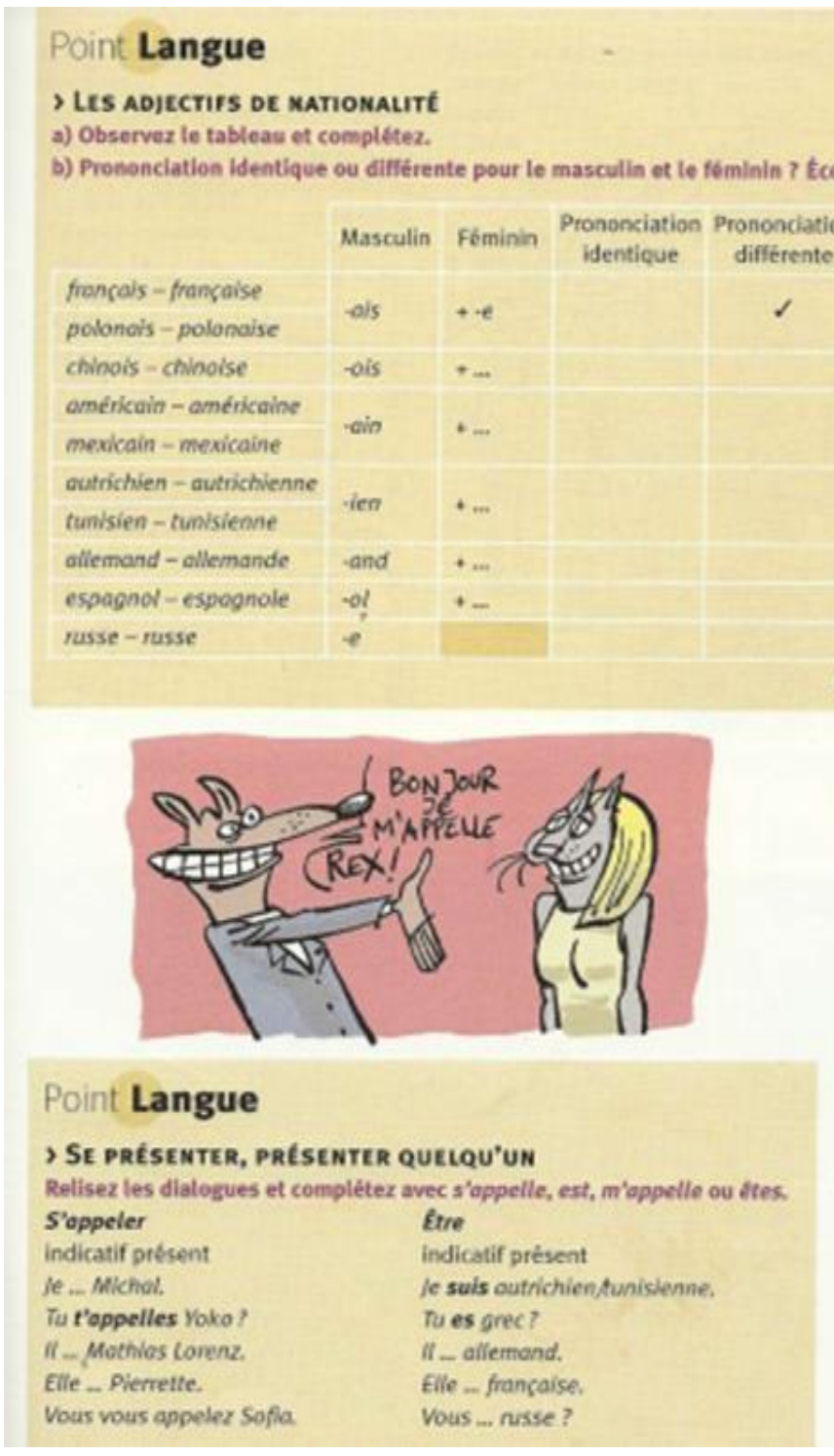

sextacen a

\section{Point Langue}

' SE PRÉSENTER, PRESENTER QUELQU'UN

Relisez les diatogues et complétez avec s'oppelle, est, m'oppelle ou étes.

S'oppeler

indicatif present

le ... Michal.

Tu t'oppelles Yoko ?

II - Mothias Lorenz.

Vous vous appeler Sofla
12 montrnaut

a) Ecouter.

b) Lisez les phrases suivantes

a volx havte.

1. Clara, c'est itatien.

2. Ivan, c'est gree?

1. Yeko, c'est japonais.

4 Bersardo, c'est espagnol.

5. Nenad, c'est suidols?

c) Trouver la fin des prénoms ot lisez-les a voix haute.

01 is

1 nad

Cla 99

Ne vas

\section{3}

\section{Jovez la scène à dewr.}

Vous étes au cocktal de bienvene.

Vous faites comaissanse.

Document 1: Alter Ego, (2006, p.13)

Cet exemple est le premier sujet de grammaire dans le livre et prend place dans la partie «point langue », autrement dit la grammaire. Le titre de ce sujet : se présenter et présenter quelqu'un. Ici ou avant sans donner aucune connaissance ou sans faire aucune explication grammaticale, à partir du dialogue abordé on demande aux apprenants d'utiliser correctement l'indicatif présent des verbes «être " et «s'appeler» dans les phrases données. On ne donne pas les conjugaisons de ces verbes avant des exercices. L'objectif est que les apprenants saisissent les règles d'emploi à l'aide des exemples précédents. Ce 
cas oblige les apprenants à faire des recherches et à construire des rapports entre les différentes parts pour constater les règles grammaticales.

Suivre une telle démarche à l'enseignement de grammaire augmente la performance individuelle et la compétence de découverte des apprenants et supprime l'apprentissage par cœur. Dans ce sens, la grammaire implicite peut être utile. Mais suivre toujours une telle démarche n'est pas convenable parce qu'il est nécessaire de temps en temps de faire des explications grammaticales. Si on vise que les étudiants trouvent les règles d'emploi, cela pourra causer à la perte du temps et à la compréhension fausse. Ainsi les apprenants peuvent atteindre en retard le niveau déterminé. Donc il n'est pas convenable de suivre toujours la même démarche.

Quand il s'agit de l'utilisation ou de l'enseignement de la langue parlée il est préférable de suivre une démarche implicite au sujet de grammaire. Car à l'utilisation de langue orale, le critère est d'être compréhensible et de réaliser la communication, le but n'est pas un usage parfait.

Pour les petits enfants et les débutants il faut préférer la grammaire implicite. Parce qu'au début ou au niveau débutant on peut apprendre les emplois simples et les règles faciles sans avoir besoin des explications.

Le plus important avantage de la grammaire implicite, c'est qu'elle oriente les apprenants aux recherches et à l'auto-apprentissage, et elle augmente leur motivation et développe leur autonomie.

\section{La grammaire explicite}

La grammaire explicite est une démarche dans laquelle on fait la description et l'explication des règles soit par le manuel, soit par l'enseignant. Ici, comme il s'agit de connaître explicitement les règles on peut parler d'un apprentissage cognitif.

Du fait qu'on apprend la langue étrangère après l'acquisition de la langue maternelle, on ne peut pas suivre le même processus avec celui de la langue maternelle. Quand l'apprenant apprend la langue cible, il connaît déjà les codes et le système de sa langue maternelle. «Les adultes en particulier, ont besoin de comprendre, ils demandent des explications, il n' y a pas d'apprentissage sans que des processus réflexifs et cognitifs ne soient pas en œuvre. » (Besse et Pourquier, 1993, p.98). C'est pour cette raison que lors de l'apprentissage d'une langue étrangère l'apprenant veut faire les comparaisons entre deux langues. Il utilise les ressemblances et les différences. Il analyse et découvert les structures de deux langues dans son esprit avant de pratiquer les règles.

Réfléchir aux règles grammaticales peut bloquer parfois le développement de la compétence orale, mais pourtant la grammaire explicite contribue à développer les compétences écrites comme la compréhension écrite et la production écrite. Le développement des compétences écrites dépend étroitement de la performance d'usage des règles de grammaire et la grammaire explicite devient un moyen avantageux pour atteindre cet objectif. Il n'est pas facile de travailler 
sur un texte difficile sans avoir des connaissances grammaticales. Pour faciliter la compréhension des apprenants il est avantageux de temps en temps de présenter le point grammatical avec des exemples, en suite de pratiquer ces connaissances sur les produits. Car chaque apprenant ne peut pas saisir facilement les règles d'emploi de la langue cible. Car au développement de ces compétences la grammaire est comme ciment à la construction d'un bâtiment. Comprendre ou construire les phrases complexes dépend de la connaissance du système de codage de la langue cible.

En mettant en relation les règles et les structures avec la fonction de la langue cible, le manuel ou l'enseignant peut jouer un rôle important dans l'enseignement de grammaire. Le but d'une telle démarche est d'aider les apprenants pour qu'ils apprennent mieux la langue cible.

Les avantages de la grammaire explicite sont les suivants:

1) Faciliter la compréhension

2) Pouvoir utiliser efficacement les structures complexes

3) Assurer confiance en soi pour mettre en œuvre les compétences.

Dans les manuels formés selon la méthode communicative et la méthode actionnelle on donne la place prioritairement à la grammaire implicite, mais de temps en temps il est possible aussi de rencontrer la grammaire explicite. « Si la grammaire relève chez l'usager à la fois de l'inné et de l'acquis, cela signifie qu'il maîtrise bien cette langue. D'ailleurs, c'est pour cette raison là qu'il n'est pas facile pour un apprenant étranger d'arriver au stade où il pourra communiquer exactement comme un natif. » (Mahommed, 2008)

Ici, nous voulons étudier un autre exemple tiré du manuel de méthode Métro Saint-Michel. C'est un livre composé à partir des principes de la méthode actionnelle, publié par la maison d'édition CLE. Le niveau de ce manuel aussi est A1 / le débutant. Voici un exemple de la partie «grammaire»: 
Günday, R. ve Ark. (2017). Quelle Demarche Suivre en Didactique de Langue Etrangere:

La Grammaire Implicite ou La Grammaire Explicite? Humanitas, 5(10), 247-262

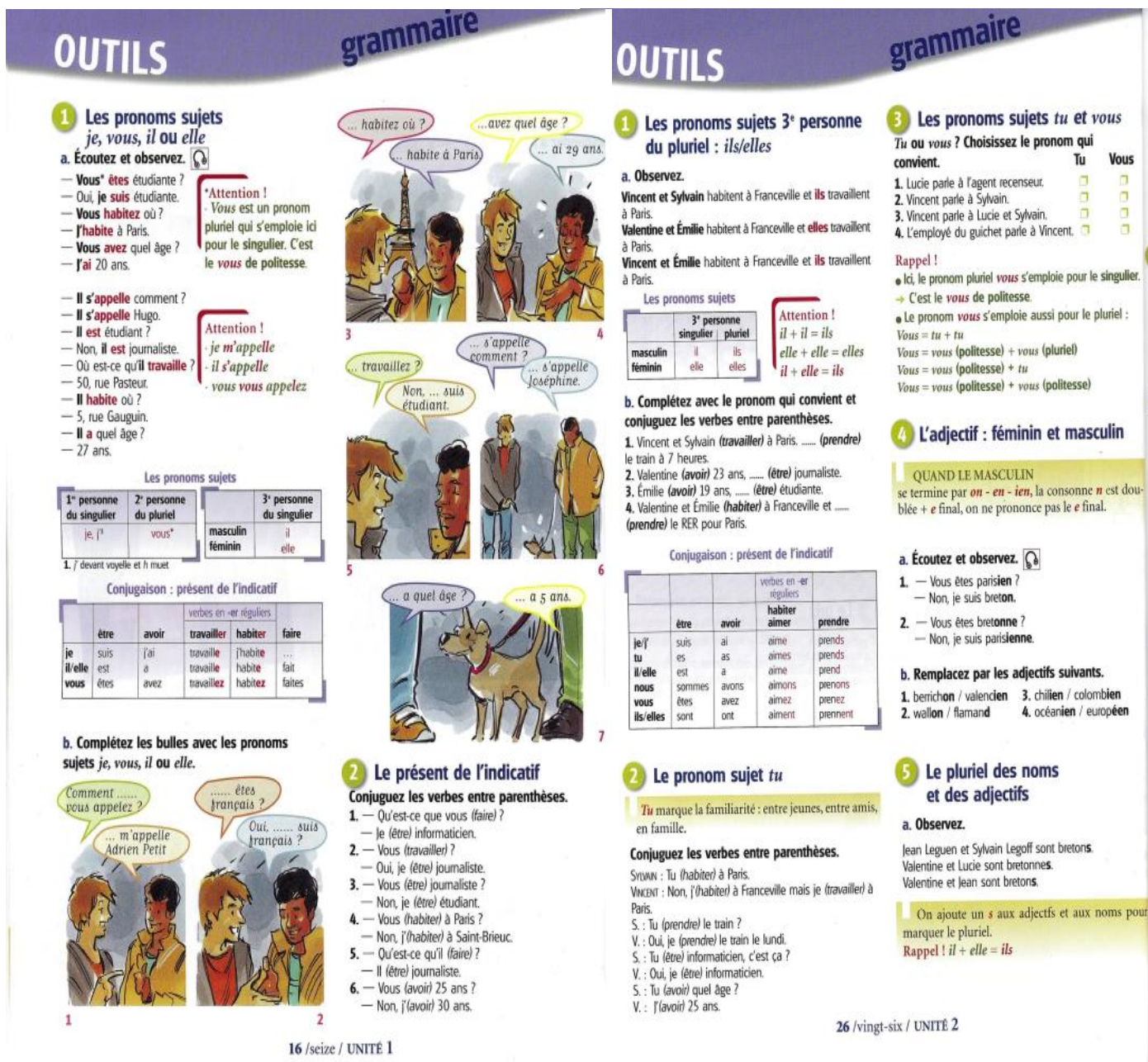

Document 2 : Métro Saint-Michel, (2006, p.26)

Cet exemple aussi, comme l'exemple dans le chapitre précédent, est le premier sujet de grammaire dans le livre et prend place dans la partie «grammaire ». Mais dans le chapitre suivant on continue à présenter le même sujet. C'est pour cette raison qu' ici nous volons citer deux exemples. Le titre du sujet: les pronoms sujets. On aborde l'emploi du présent de l'indicatif. Dans le premier exemplaire on conjugue le présent de l'indicatif de cinq verbes (être, avoir, travailler, habiter, faire). Dans le deuxième exemplaire on conjugue le présent de l'indicatif de quatre verbes (être, avoir, aimer, prendre). Dans ce livre aussi le premier verbe est «être ». Au début on donne la conjugaison des verbes dans un tableau, puis on passe aux exercices. Autrement dit, d'abord on présente la connaissance ou fait des explications grammaticales, ensuite on demande aux apprenants de faire les exercices dans lesquels se trouvent les verbes enseignés plus avant. L'objectif est que les apprenants utilisent consciemment ces verbes 
dans les exercices. On vise à aider les apprenants pour qu'ils apprennent clairement et sans perdre le temps. Dans ce manuel on présente plusieurs verbes en même temps, dans ce cas sans faire des explications il est difficile de les utiliser correctement.

S'il faut donner des exemples de manuels écrits en Turquie sur l'enseignement du FLE, Je parle Français et Je voyage en Français contiennent des démarches soit implicite, soit explicite. Des dialogues et des textes des manuels contiennent "des situations" qui ont été utilisées dans la vie courante. Le professeur, en faisant appel au geste et aux mimiques, présente les situations et enseigne le français. À l'aide des situations, les élèves peuvent comprendre le sens et la structure des répliques des dialogues et en les imitant ou en les répétant, ils peuvent essayer de prendre la parole facilement dans les activités en classe. Ainsi, les élèves peuvent apprendre implicitement plusieurs règles grammaticales en profitant des expressions. Par exemple, les élèves avaient déjà appris l'expression «Qu'est-ce que c'est ? » Lors de la leçon le professeur peut entendre un bruit, et dans une intonation interrogative, il peut dire: "Qu'est-ce que c'est ? », Les élèves répondent : «C'est un camion». Puis, le professeur peut reprendre la parole et peut poser cette question: «C'est un camion qui passe à côté de l'école ? » et les élèves lui répondent à nouveau : " Oui, c'est un camion qui passe à côté de l'école » ou « Non, ce n'est pas un camion qui passe à côté de l'école, c'est un train qui passe à côté de l'école » (Kuşçu, 2016, pp.111-113). A l'aide de ces conversations, les élèves peuvent apprendre implicitement et facilement une des règles grammaticales du français. Mais dans ces manuels il est possible aussi de voir de temps en temps des exemples de la grammaire explicite. Par exemple dans Je voyage en Français (Üstün et all, 1997, p.102) lors qu'on enseigne les pronoms on suit une démarche explicite. D'abord on présente le tableau des pronoms, puis on donne des exemples.

Bref, s'il faut faire un résumé de considération: «alors que la grammaire implicite met l'accent sur l'aspect indirect de l'enseignement/apprentissage de la grammaire d'une langue cible, la grammaire explicite, s'intéresse à un métalangage original et simplifié dans l'enseignement/apprentissage d'une langue étrangère. Il n'est pas tout à fait vrai de dire que la grammaire explicite relève de la grammaire de référence (où on s'intéresse à l'aspect descriptive de la grammaire), alors que la grammaire implicite appartient à la grammaire pédagogique (où le point est mis sur l'usage pour arriver à l'aspect descriptive de la grammaire). » (Mahommed, 2008). En outre, alors que la grammaire implicite vise à donner aux apprenants la maitrise d'un fonctionnement grammaticale et à s'appuyer sur une manipulation, la grammaire explicite est fondée sur l'explication des règles par le manuel ou l'enseignant où il s'agit des applications conscientes par les apprenants et de travailler sur le métalangage (Coste et Galisson, 1976, pp.206, 245).

L'importance de la grammaire explicite varie selon les niveaux d'apprentissage. Tant que les niveaux s'avancent et lorsqu'il s'agit des règles complexes, des 
textes ou des productions écrites au niveau avancé on a besoin de la riche connaissance de grammaire, dans ce cas il est utile de faire des explications grammaticales et de suivre s'il est nécessaire la grammaire explicite. "Aux niveaux avancé et supérieur les apprenants ont besoin de précision et apprécient de faire le point complet sur une question grammaticale, alors que des débutants et des faux-débutants n'ont pas toujours les moyens d'assimiler l'explication complète. » (Fougerouse, 2001, p.7). Bien qu'on suive en général la grammaire implicite, selon les niveaux d'apprentissage des apprenants ou la difficulté du sujet, il est possible de choisir la grammaire implicite ou la grammaire explicite.

\section{Conclusion}

Pour pouvoir pratiquer efficacement les compétences langagières on a besoin de connaître la structure fonctionnelle de la langue cible. La bonne maîtrise de la grammaire de langue étrangère influence positivement le développement de toutes les compétences. S'il faut considérer la langue comme un système de codes, la connaissance de grammaire est la clé de ce système. Les messages sont construits sur ce système, c'est-à-dire la production et la compréhension dépendent de l'utilisation de façon exacte de ce système qui couvre la grammaire aussi. Quand il s'agit de la réalisation d'une communication, d'une tâche, d'une action orale ou écrite il est inévitable d'avoir une connaissance linguistique.

Pourtant distinguer une grande partie des activités, autrement dit du cours, à la grammaire n'est pas convenable, parce que la concentration excessive sur la grammaire peut empêcher le développement des compétences principales. Mais il n'est pas négligeable tout à fait la place de la grammaire dans l'enseignement de la langue étrangère. Il faut avoir recours à l'enseignement de grammaire s'il est nécessaire et suivre la démarche la plus efficace.

$\mathrm{Au}$ niveau débutant et avec les petits il est utile de suivre une grammaire implicite, au niveau avancé lorsqu'on étudie les sujets complexes et difficiles on peut préférer une grammaire explicite. Donc la démarche à suivre dépend de certains critères. Ce qui est important dans l'enseignement de grammaire, c'est de suivre une démarche convenable aux besoins et niveaux des apprenants.

\section{Bibliographie}

Berthet, A. et all. (2006). Alter Ego, Paris: Hachette.

Besse, H. (1992). Méthodes et pratiques des manuels de langue. Paris : Didier.

Besse, H. et Porquier, R. (1993). Grammaires et didactiques des langues, Paris : Hatier/Crdif.

Bozbeyoğlu, S. (2000). Enseignement du Français Langue Etrangère. Ankara : Şahin Matbaası.

Chartrand, S-G. (1996). Pour un nouvel enseignement de la grammaire, Canada : Les Editions Logiques. 
Günday, R. ve Ark. (2017). Quelle Demarche Suivre en Didactique de Langue Etrangere: La Grammaire Implicite ou La Grammaire Explicite? Humanitas, 5(10), 247-262

Courtillon, J. (1989). La grammaire sémantique et l'approche communicative, dans «...Et la grammaire? ». Le Français dans le monde/Recherches et applications, Paris: Hachette EDICEF, Février-Mars 1989, 118-127.

Coste, D. et Galisson, R. (1976). Dictionnaire de didactique des langues, Paris : Hachette.

Erbaş, A. et Tanrıkulu, H. (1994). Je parle Français, İstanbul: Milli Eğitim Basımevi.

Fougerouse, M.C. (2001). L'Enseignement de la Grammaire en Classe de Français Langue Etrangère, Revue Ela, 2-16.

Germain, C. et Seguin, H. (1995). Le point sur la grammaire en didactique des langues, Canada: CEC.

Günday, R. (2015). Yabancı dil ögretiminde yaklaşımlar, yöntemler, teknikler ve multimedya araç ve materyalleri. Ankara: Favori Yayınları.

Kuşçu, E. (2016). Les méthodologies et les méthodes d'enseignement/apprentissage du FLE en Turquie: "Je parle Français et Je voyage en Français", International Journal of Languages' Education and Teaching (Ijlet). Issue 2, December 2016, 105-121.

Mahommed, A. (2008). Faut-il ignorer complètement la grammaire explicite en FLE? Colloque Bruxelles, 20-22 Mars 2008.

Monnerie-Goarin, A. et all. (2006). Métro Saint-Michel, Paris, CLE.

Robert (2015). Dictionnaire français.

Üstün, O. et all. (1997). Je voyage en Français, İstanbul: Milli Eğitim Basımevi.

Vigner, G. (2001). Enseigner le Français comme langue seconde, Paris: CLE.

\section{WHICH METHOD SHOULD BE USED IN FOREIGN LANGUAGE TEACHING: THE IMPLICIT GRAMMAR OR THE EXPLICIT GRAMMAR?}

Abstract: There is a close relationship between knowledge of language and effective use of language. It is impossible to discover a language's syntactic, structural and semantic properties without knowing its grammar and it is difficult to develop language skills without having mastered it enough. If the language is considered as a system, it is necessary to identify the codes of this system in order to understand it and produce in it. It is inevitable to know the use of grammar rules in order to be able to use elements of the sentences correctly, to communicate correctly, to express oneself verbally and especially in written form. Grammar maintains its indispensable place in foreign language teaching even if the importance of grammar teaching decreases from time to time or changes from one method to another. Eight different ways are followed to teach the grammar of the target language: implicit and explicit grammar, inductive and deductive grammar, contextual and out-of-context grammar, active and passive grammar. Grammar can also be handled in 
Günday, R. ve Ark. (2017). Quelle Demarche Suivre en Didactique de Langue Etrangere:

La Grammaire Implicite ou La Grammaire Explicite? Humanitas, 5(10), 247-262

terms of word, spelling, sentence and text. In this study we will discuss implicit grammar and explicit grammar. Implicit grammar teaching aims to teach learners how to use the target language without applying to theoretical knowledge and grammar explanations. When such a course is followed, it is an intuitive learning. In the case of explicit grammar teaching, a way in which rules are described and explained by a coursebook or teacher is followed. In this type of teaching, there is cognitive learning because the rules are openly recognizable. In this study, we will also discuss the role that explicit grammar and implicit grammar play in the development of language skills. As a result, we will look for an answer to the question of which way is more effective in foreign language learning.

Keywords: foreign language, teaching, way, explicit grammar, implicit grammar. 